BULLETIN Bulletin hispanique

HISPANIQUE Université Michel de Montaigne Bordeaux

119-2 | 2017

La Égloga renacentista en el Reino de Nápoles

\title{
El grupo poético de Ischia y la adaptación al vulgar de la égloga piscatoria
}

\section{Gáldrick de la Torre Ávalos}

\section{(2) OpenEdition \\ 1 Journals}

\section{Edición electrónica}

URL: http://journals.openedition.org/bulletinhispanique/5079

DOI: 10.4000/bulletinhispanique.5079

ISSN: $1775-3821$

\section{Editor}

Presses universitaires de Bordeaux

\section{Edición impresa}

Fecha de publicación: 1 diciembre 2017

Paginación: 537-554

ISBN: 979-10-300-0218-8

ISSN: 0007-4640

\section{Referencia electrónica}

Gáldrick de la Torre Ávalos, «El grupo poético de Ischia y la adaptación al vulgar de la égloga piscatoria», Bulletin hispanique [En línea], 119-2 | 2017, Publicado el 28 diciembre 2020, consultado el 15 enero 2021. URL: http://journals.openedition.org/bulletinhispanique/5079 ; DOI: https://doi.org/ 10.4000/bulletinhispanique.5079 


\title{
El grupo poético de Ischia y la adaptación al vulgar de la égloga piscatoria
}

\author{
GÁldrick de la Torre Ávalos \\ Universitat de Girona
}

Dans son commentaire de la poésie de Bernardo Tasso, Fortunato Pintor émet l'hypothèse connue selon laquelle l'accès au vulgaire de l'églogue piscatoire a eu lieu en 1533 à Ischia, à la demande de la marquise de Pescara. Il se fonde, entre autres, sur le témoignage de Scipione Ammirato, qui fait de Berardino Rota l'initiateur de la piscatoire en langue toscane. Bien que Rota ait publié tardivement ses piscatoires, de nombreux indices corroborent l'affirmation d'Ammirato. Le présent article en approfondit certains.

Mots-clés: Bernardo Tasso, Berardino Rota, Alfonso d'Ávalos, églogue piscatoire, cénacle d'Ischia.

Fortunato Pintor, en su comentario de la lírica de Bernardo Tasso, plantea la conocida hipótesis de que la adaptación al vulgar de la égloga piscatoria tuvo lugar en Ischia en el año 1533, a instancias de la marquesa de Pescara; lo hace basándose, entre otros, en el testimonio de Scipione Ammirato, quien asigna a Berardino Rota el papel de introductor de la piscatoria en la lengua toscana. A pesar de que Rota publica sus piscatorias de forma tardía, existen numerosos indicios para pensar que la afirmación de Ammirato es cierta. El presente artículo ahonda en algunos de ellos.

Palabras clave: Bernardo Tasso, Berardino Rota, Alfonso d'Avalos, égloga piscatoria, cenáculo de Ischia.

In his commentary on the poetry of Bernardo Tasso, Fortunato Pintor proposed the well-known hypothesis that access of the piscatory eclogue to the common took place in Ischia in 1533, on the Marchioness of Pescara's order. He based this idea on, among others, Scipione Ammirato's testimony — he who assigned to Berardino Rota the role of introducing the piscatory into the Tuscan language. Despite the fact that Rota published his piscatorial works late on, numerous indications suggest that Ammirato was right. This article goes deeper into some of them.

Keywords: Bernardo Tasso, Berardino Rota, Alfonso d'Ávalos, piscatory eclogue, Ischia circle. 
$\mathrm{E}$ n 1560, pocos meses antes de la publicación de las Egloghe pescatorie, el editor napolitano Scipione Ammirato imprimía los Sonetti in morte que Berardino Rota había dedicado a su difunta esposa Porzia Capece ${ }^{1}$. En la carta introductoria de este su primer cancionero, Ammirato le explicaba a Annibal Caro que, de no ser por él, no habría podido publicar las rimas, ya que, al enviudar, Berardino había dejado de interesarse en la publicación de su obra ${ }^{2}$. Por ello le daba las gracias y le enviaba un ejemplar de este volumen. Annibal no solo había conseguido que Rota le diese permiso a Ammirato de publicar las rimas dedicadas a la memoria de su mujer, sino que en breve Ammirato podría sacar a la luz un volumen con las piscatorias y otros poemas dedicados a la vida y a la muerte de la dama, además de parte de su producción poética latina. Lo relevante de todo ello es que ya entonces Ammirato declara a Berardino ser «il primiero inuentore» de la égloga piscatoria en lengua toscana, así como lo fue Teócrito en la lengua griega y Sannazaro en la latina.

Esta es una idea que se repite también en la publicación de las piscatorias ${ }^{3}$. En este caso el editor añadía además algunos datos relativos al contexto en que se gestaron. En la carta que dirige a Giovan Francesco Mormile, Ammirato escribe lo siguiente:

V'envio dunque questo nuovo presente, il quale mi persuado sia per dovervi esser caro, si per esser parto d'un amico vostro e d'un gentil'uomo della vostra patria, e si per esser opera giovenile e conveniente eziandio da questa parte all'età di voi, poi che già sono xxvii anni ch'egli vi pose mano, et ebbene per ascoltatrice Vittoria Colonna marchesana di Pescara di buona memoria: a cui piacquero cotanto queste egloghe per la lor vaghezza e per li molti lumi e bellezze di che elle sono ripiene, che n'avea gran parte a memoria, e recitavale e celebravale come frutto di sommo poeta et illustre, tutto che in assai breue spazio di tempo fussero da lui state dettate e composte. ${ }^{4}$

Teniendo en cuenta que en la carta se afirma que hace 27 años que escribió las piscatorias y que la carta es del 15 de julio de 1560, así como el poco tiempo que Berardino empleó en su composición, según el mismo Ammirato Rota habría escrito sus piscatorias en el año 1533.

1. El presente trabajo forma parte del Proyecto de Investigación FFI2015-65093-P ( GGarcilaso de la Vega en Italia. Estancia en Nápoles») financiado por el Ministerio de Economía y Competitividad.

2. Cf. Berardino Rota, Sonetti del s. Berardino Rota in morte della s. Portia Capece sua moglie, Napoli, Mattia Cancer, marzo de 1560, «Al sig. Anibal Caro», fol. A ii.

3. "Già si vede chiaramente a che altezza et a che sommità vada crescendo la bellissima nostra lingua toscana: poi che non solo si vede tuttavia riempire di prosatori e di poeti di grande stima e di conto, ma eziandio di varie sorti di poemi. In guisa che non solo di nulla cede alla lingua latina, ma di gran lunga da questo lato la supera e le va avanti, come oggi potete veder con l'Egloghe pescatorie del Signor Berardino Rota, primiero inventore di esse egloghe in questa lingua». Se cita a partir de la moderna edición de Stefano Bianchi, basada en la prínceps napolitana de Maria Scotto, 1560: Berardino Rota, Egloghe pescatorie, ed., introd. y notas Stefano Bianchi, Roma, Carocci, 2005, p. 75 .

4. Ibid. 
Entrado el siglo XX Fortunato Pintor recogía este testimonio para formular su hipótesis de que fue en ese año, en Ischia, cuando Berardino Rota habría compuesto sus piscatorias a instancias de la marquesa de Pescara Vittoria Colonna $^{5}$. A juicio de Pintor, la marquesa habría instado al también poeta Bernardo Tasso y a Berardino Rota a practicar en lengua vulgar lo que Sannazaro había llevado a cabo con la égloga neolatina -esto es, trasladar el código pastoril al escenario piscatorio de la Bahía de Nápoles y de sus mitos-; y todo ello en una especie de justa poética que habría tenido lugar en la isla y de la que habría salido victorioso el poeta napolitano.

Ahora bien, el hecho de que Pintor se base en el testimonio de Ammirato, que, como es sabido, además del editor de Berardino Rota, era también su amigo, nos obliga a preguntarnos si la afirmación de Ammirato es cierta y no se trata realmente de una operación destinada a convertir a Berardino Rota en el introductor de la piscatoria en la lengua toscana. Ya hemos visto la insistencia con la que el editor buscaba atribuirle este papel. Por otro lado, sabemos que por las mismas fechas en que el poeta compuso sus piscatorias, salvando las distancias, que nacen de un alejamiento con el modelo sannazariano, el conde Matteo di San Martino escribía su Pescatoria et egloghe (Venezia, Giolito 1540) y Girolamo Britonio el Elegantissimo dialogo pastorale et maritimo (Roma, Blado 1535), obras que incluso llegaron a publicarse con anterioridad a las de Berardino Rota ${ }^{6}$. Por todo ello, se impone la necesidad de confirmar si es el testimonio de Scipione Ammirato un testimonio verídico y si es, en efecto, en 1533, en Ischia, cuando y donde se produjo la adaptación al vulgar de la égloga piscatoria.

El punto de partida de nuestra investigación, dadas las palabras de Ammirato y de su ampliación en el comentario de Pintor, no puede ser otro que el del cenáculo de Ischia, dentro del cual, en teoría, Berardino habría compuesto sus piscatorias ${ }^{7}$. Un buen reflejo del momento en que se produce tal acontecimiento

5. Cf. Fortunato Pintor, Delle liriche di Bernardo Tasso, Pisa, Tipografia Successori Fratelli Nistri, 1900, p. 114 y ss. El libro es un extracto de la obra publicada con el mismo título, también ese año, en los Annali della R. Scuola Normale Superiore di Pisa: Filosofia e Filologia, vol. XIV.

6. Cf. Domenico Chiodo, «Le Pescatorie del Rota tra egloga e idillio», en Suaviter Parthenope canit. Per ripensare la 'geografia e storia' della letteratura italiana, Soveria Mannelli, Rubbettino Editore, 1999, pp. 70-71; Stefano Bianchi, «Introduzione», en Berardino Rota, op. cit, pp. 9-15.

7. Sobre el cenáculo de Ischia, cf. Suzanne Thérault, Un cénacle humaniste de la Renaissance autour de Vittoria Colonna, châtelaine d'Ischia, Firenze, Edizioni Sansoni Antiquariato, 1968; Tobia R. Toscano, "Due "allievi" di Vittoria Colonna: Luigi Tansillo e Alfonso d'Avalos», Critica letteraria, $\mathrm{n}^{\circ}$ 16, 1988, pp. 739-773; Ippolita Di Majo, "Il castello di Ischia e la cultura delle corti», en Pina Ragioneri (ed.), Vittoria e Michelangelo. Catalogo della mostra a Casa Buonarroti, 24 maggio-12 settembre 2005, Florence, Mandragora, 2005, pp. 19-32; Concetta Ranieri, "Vittoria Colonna e il cenacolo ischitano», en M. Santoro (ed.), La donna nel Rinascimento meridionale. Atti del convegno internazionale, Roma, 11-13 novembre 2009, Pisa-Roma, Fabrizio Serra, 2010, pp. 49-62; Mauro Marrocco, «Ischia e il suo cenacolo di primo Cinquecento: un rinnovato Parnaso per le muse meridionali», en B. Alfonzetti, G. Baldassarri y F. Tomasi (eds.), I cantieri dellitalianistica. Ricerca, didattica e organizzazione agli inizi del xxi secolo. Atti del XVII congreso dell' ADI-Associazione degli Italianisti (Roma Sapienza, 18-21 settembre 2013), Roma, 
lo tenemos en el Dialogo de Giovio, cuyos hechos, a pesar de que se remontan a finales de 1527, dada la presencia de algunos anacronismos, resultado de los distintos periodos de redacción de la obra, extenderían su validez histórica hasta 1535, motivo por el que resulta un buen testimonio de la recepción napolitana y en particular ischitana de la cultura literaria y artística de aquel tiempo ${ }^{8}$. Allí, además de referir a Berardino como digno discípulo de su maestro Marc'Antonio Epicuro, al comienzo del libro II, en que se menciona la figura de Rota, se establece una suerte de comparación entre Sannazaro, que constituye la autoridad local, con su homólogo fuera de Nápoles Pietro Bembo. Es dentro de esta analogía, que compara tanto en latín como en vulgar la obra de los dos escritores, donde se menciona la reciente publicación del De partu Virginis y con ella también la de las piscatorias, puesto que ambas salieron de la imprenta de Antonio Frezza en un mismo volumen un año antes, en 1526. Quien habla es Giovan Antonio Muscettola, que, delante de la estatua de Pontano, en la isla de Ischia, se dirige a sus dos contertulios Alfonso d'Ávalos y el mismo Paolo Giovio añadiendo lo siguiente:

Piscatoriae vero et peramoena tituli novitate, et varietate maritimarum rerum, et suavitate carminum adeo sunt admirabiles, ut multorum iudicio nullis vel antiquorum operibus cedant. ${ }^{9}$

El fragmento es un indicio de la recepción napolitana de las piscatorias de Sannazaro, que gustaron no solo por la novedad del título y la dulzura de las canciones, que también podía encontrarse en los poemas de la Arcadia, sino por la variedad temática que entrañaba la propia materia marítima. Quizá sea este el motivo por el que años más tarde también Giovio, en los Elogia, vuelve sobre las piscatorias para añadir que tuvieron una mayor acogida incluso que el De partu Virginis:

Gravi autem et sacro poëmate "De partu Virginis", viginti annorum lima perpolito, summum decus frustra expectasse videri potuit, quum illae quae iuveni exciderant "Piscatoriae eclogae» publico exceptae plausu reliquorum operum famam oppresserint, ita ut eam publici tanquam iniqui iudicii querelam aperto cum pudore nec tamen sine tacita voluptate devoraret. ${ }^{10}$

Prueba del interés existente en las tertulias de Ischia por las piscatorias de Sannazaro, además del ya citado Dialogo de Giovio, lo constituye el hecho de

Adi editore, 2014, pp. 1-7.

8. En lo que respecta a la presencia de anacronismos en el Dialogo, parecen estar de acuerdo sus dos principales editores en la actualidad; a saber, Franco Minonzio, cuya edición sirve de base para el presente estudio, y Kenneth Gouwens, su primer traductor al inglés: cf., respectivamente, Paolo Giovio, Dialogo sugli uomini e le donne illustri del nostro tempo, Torino, Aragno, 2011, 93-94, passim; y Notable Men and Women of Our Time, Cambridge, The I Tatti Renaissance, 2013, p. 10.

9. Paolo Giovio, op. cit., p. 194.

10. Paolo Giovio, Elogia virorvm illvstrivm, s.v. Iacobus Sanazarius; en Opera, Roma, Istituto Poligrafico dello Stato, 1972, t. VIII, p. 104. 
que uno de los ejemplares de lujo que se conservan de esta primera edición de la obra, el ejemplar G 10031 de la British Library de Londres, contiene en el fol. A4r lo que parece un emblema de la marquesa de Pescara ${ }^{11}$. Dicho emblema está formado por una columna de color gris, sobre fondo rojo, con un pedestal y un capitel dorados a cuyos lados puede leerse, transcritas en oro, respectivamente, las iniciales $\mathrm{V}$ y C; lo que indicaría que se trata, con toda probabilidad, de un ejemplar que perteneció a Vittoria Colonna. En el contexto en que se escribe el Dialogo de Giovio, la invasión del mariscal Lautrec y de la peste en Nápoles, es decir, después de que se hicieran públicas sus piscatorias, Sannazaro habría estado en Ischia siguiendo a los otros ciudadanos que huían de la guerra y de la peste ${ }^{12}$. Es posible, como anotan Fantazzi y Perosa, quienes describen el ejemplar, que el poeta hubiera querido con él «fare omaggio di una copia a stampa, lussuosamente decorata, del suo poema», refiriéndose al De partu Virginis, pero también, añadimos, a las piscatorias ${ }^{13}$.

Dentro del entorno poético de Vittoria Colonna, también Berardino Rota poseía uno de los manuscritos que se conservan del De partu Virginis, el cual, además, trae lecciones interesantes por cuanto se trata de una de las copias que Sannazaro había utilizado para la imprenta. Es el mismo caso que el de su amigo Alfonso Cambi, con quien aparece representado literariamente en el diálogo Il Rota overo dellimprese, escrito por Scipione Ammirato, y quien poseyó también una de estas copias probablemente por herencia paterna. Si tenemos en cuenta que su padre, Tomaso Cambi, era el secretario de Vittoria Colonna por las mismas fechas en que Sannazaro estuvo en Ischia, nada impide pensar, por lo tanto, que fuera la propia marquesa de Pescara quien le hiciera entrega del manuscrito, lo que nos conduciría, una vez más, al epicentro de estas relaciones ${ }^{14}$.

Curiosamente, en la única carta que se conserva de la relación entre Vittoria Colonna y Berardino Rota, la cual se incluye en el epistolario de Ferrero y Müller, aparece también mencionado Tomaso Cambi ${ }^{15}$. Si bien la carta carece de fecha, es bastante probable que Vittoria Colonna la escribiera durante el tiempo que permaneció en Ischia, entre 1527 y 1534, en especial durante sus últimos años ${ }^{16}$. En ella Cambi aparece representado como el encargado

11. Cf. Charles Fantazzi y Alessandro Perosa, «Introduzione», en Iacopo Sannazaro, De partu Virginis, Firenze, Leo S. Olschki Editore, 1988, pp. XLVIII y XLIX.

12. Cf. Minonzio, en Paolo Giovio, op. cit., vol. II, n. 17.

13. Fantazzi y Perosa, op. cit, p. XLIX.

14. Se trata, respectivamente, de los códices denominados $A$ y $L$, los cuales se encuentran en la Biblioteca Medicea Laurenziana y aparecen descritos también en la introducción de Fantazzi y Perosa al De partu Virginis; cf., op. cit., pp. XIV-XX.

15. Cf. Vittoria Colonna, Carteggio, $2^{\mathrm{a}}$ ed., con suplemento de Domenico Tordi, ed., introd. y notas Ermanno Ferrero y Giuseppe Müller, Torino, Ermano Loescher, 1892, carta núm. LX, pp. 90-91. Toscano comenta también esta carta, «databile al 1532-33», en op. cit, pp. 747-748; cf. también Di Majo, op. cit, p. 28.

16. Sobre la cronología de Vittoria Colonna en Ischia, cf. Thérault, op. cit., cap. IV «Les jeunes châtelains d'Ischia», así como Toscano, op. cit., donde relaciona el periodo de actividad del cenáculo de Ischia con la labor mecénica de Alfonso d’Ávalos, y mi propuesta de periodización 
de hacer llegar la carta al hermano de Berardino, quien teóricamente debiera entregársela en mano. La marquesa empieza disculpándose por los «milli fastidii» a los que tiene acostumbrados al poeta y le pide que le encargue a un «mastro» de la ciudad que le construya una «cascetta... ad modo de coliseo, tutto a colonnati bianchissimi, et le corone, capitelli et intorno e tutto, dove se pò, molto doraton, lo que nos recuerda el gusto humanístico de la época y también el ya citado emblema de Vittoria Colonna. A partir de ahí, solo añade que la caja debería contener unos "profumi mediochi, ma lavorati bianchi» junto con unos "carrafelle, et altro, che forsi se trovaranno fatti»; todo al gusto del «sig. Marchese» Alfonso d'Ávalos, que es quien aparentemente hace el pedido ${ }^{17}$ : la marquesa de Pescara, por su parte, es quien coordina y delega la tarea en Berardino Rota, que se encarga con «il mio Epicuro» (Marc'Antonio) de componer los epigramas que deben aparecer entre las dos columnas. En definitiva, una epístola de corte anecdótico que, si bien ofrece datos de escaso interés para el contexto, sí que nos ayuda a vislumbrar siquiera el ambiente cortesano de la época y el alcance de las relaciones de Vittoria Colonna, que parece mantener una relación amigable tanto con Berardino Rota como con su maestro Marc'Antonio Epicuro ${ }^{18}$.

Más interesante para nuestro propósito es el libro de églogas de Berardino Rota. A imitación de las piscatorias sannazarianas, que parten del modelo conjunto de Virgilio y de Teócrito, en muchas de ellas aparecen toda una serie de reflexiones metapoéticas que resultan apropiadas por cuanto nos indican mediante el alegorismo propio de la égloga algunas circunstancias relativas a su proceso de composición. Por ejemplo, la égloga número VIII, en la que Licidas, el alter ego del poeta, parafraseando a Licón en la segunda égloga de Sannazaro ${ }^{19}$, declara haber recibido el "pregio» de la "rete toscana» -esto es, de las piscatorias- de esa «nova Safo» que tiene el poder de amansar a las

en Gáldrick de la Torre Ávalos, “"... al servitio de la felice memoria del Marchese del Vasto”. Notas sobre la presencia de Bernardo Tasso en la corte poética de Ischia», Studia Aurea, vol. 10, 2016, p. 364 y ss.

17. Era conocido el gusto del marqués del Vasto por los artículos de lujo; cf. Pietro Aretino, Un pronostico satirico, ed. Alessandro Luzio, Bergamo, Istituto Italiano d'Arti Grafiche, 1900, passim. El mismo Luzio cita en su edición (n. 1. 93, p. 55) un soneto también de Aretino, ya publicado por Trucchi (Poesie, III, 212) y reimpreso en los Manoscritti it. della Bibl. Nazionale di Firenze, I, 255, que alude a la afición del marqués por los perfumes y otros accesorios de baño: «Il marchese del Vasto havea pensato...». Sobre el coleccionismo de Vittoria Colonna y en general de la familia d'Ávalos, cf. Di Majo, op. cit.

18. Fruto también de esta relación son los epigramas X y LIX que Berardino Rota dedica a Vittoria Colonna en el Epigrammatum liber; cf. Berardino Rota, Carmina, ed., introd. y notas Cristina Zampese, Torino, Res, 2007.

19. "... Tingenda tibi iam lana paratur / qua niteas superesque alias, Galatea, puellas, / lana maris spumis quae mollior. Hanc mihi pastor / ipse olim dedit, hanc pastor Melisaeus, ab alta I cum me forte senex audisset rupe canentem" (Iacopo Sannazaro, Latin Poetry, ed., trad. y notas Michael C. J. Putnam, Cambridge, The I Tatti Renaissance, 2009, égloga II «Galatea», p. 114, vv. 39-43). Los versos se remontan, en última instancia, a la segunda bucólica de Virgilio: «Est mihi disparibus septem compacta cicutis / fistula, Damoetas dono mihi quam dedit olim» (Virgilio, Bucólicas, ed., trad. y notas Vicente Cristóbal, Madrid, Cátedra, "Letras Universales», 1996, p. 98, vv. 36-37). 
fieras y de tranquilizar las aguas con su decir "dolce e sovrano». Se trata, por supuesto, de Vittoria Colonna, quien recibe el nombre de Nice o de Safo según la denominación con la que otros poetas de aquel tiempo se referían poéticamente a la marquesa de Pescara ${ }^{20}$. Este es uno de los argumentos que utiliza Pintor para defender su hipótesis de que Vittoria Colonna habría sido la responsable de la adaptación de la piscatoria a la lengua toscana. Ahora bien, el hecho de que Licidas haga referencia al "pregio» y no al "premio", como cabría esperar en caso de que se estuviese aludiendo a una supuesta justa poética entre Berardino Rota y Bernardo Tasso, a nuestro modo de ver pondría ciertos límites a la hipótesis de Pintor, que, por otro lado, saldría reforzada si consideramos, en primer lugar, la identidad de los personajes que aparecen en la obra de los dos escritores y si tenemos en cuenta el hecho de que, por las mismas fechas, Giovio escribió su famoso Dialogo, en su caso declaradamente a instancias de la marquesa de Pescara, y que parece ser también durante este tiempo cuando el De poeta de Minturno recibió su impulso definitivo ${ }^{21}$.

Pero donde mejor puede apreciarse hasta qué punto las piscatorias de Berardino Rota están en deuda con la marquesa de Pescara es, sin duda, en la égloga IX. A diferencia de la anterior, donde la presencia de Vittoria se reduce a un comentario de pasada y en donde Ischia aparece como un elemento más de la Bahía de Nápoles, en esta égloga el escenario del poema es la isla y su protagonista Vittoria Colonna, de quien Timeta y Meliseo, además del propio poeta, celebran su natalicio. Es, por tanto, una composición que entra dentro del género genethliacon, lo que, de alguna manera, prefigura el fondo cortesano de la obra. De entrada, el poema llama la atención por su parecido formal con la égloga primera, que abre el conjunto del libro. En esta otra el poeta invoca a las ninfas de Mergellina, como hace Virgilio con las musas sicélides en la cuarta de sus bucólicas, para que le ayuden a seguir las huellas del pescador Sannazaro (Licón), a quien tuvieron el gusto de escuchar durante el tiempo que vivió en Mergellina. El poeta, que también en este caso podría ser Licidas, abandona "gli orti aprici e cari / de la bella Egla» (vv. 19-20), que es el nombre

20. Con el nombre de Nice, que en griego significa 'victoria', aparece representada en la égloga homónima de Marco Girolamo Vida y en los poemas de Niccolò d’Arco y de Marcantonio Flaminio (cf. Leonard W. Grant, Neo-Latin Literature and the Pastoral, Chapel Hill, University of Carolina Press, 1965, cap. X, p. 314). Por su parte, Bernardo Tasso la compara con Safo en la carta dedicatoria que abre el conjunto de églogas y elegías de su segundo libro de los Amori: «Sperando che, si come sola, quell'altissimo grado di perfezione che in ciascun'arte et in ogni scienza si ritrova, occupato tenendo, Safo e tutte l'altre nelle bone lettere più famose di gran lunga avanzando, e col volo delle vostre proprie penne sopra le stelle levandovi, avete co' raggi della vostra virtù illustrata questa nostra età, sarete eziandio contenta che queste mie egloghe et elegie vivino nel seno della vostra gloria...."

21. En lo que respecta al patrocinio de Vittoria Colonna, el mismo Giovio se refiere a él al comienzo de su diálogo: "cohortante Victoria dialogum conscripsi» (cf. op. cit., pp. 8-9). En cuanto a la relación del De poeta de Minturno con el cenáculo de Ischia, cf. el reciente estudio de Eugenia Fosalba: «Tracce di una precoce composizione (ca. 1525-1533) del De Poeta di Minturno. A proposito della sua possibile influenza su Garcilaso de la Vega», Critica letteraria, no 173,2017 , pp. 627-650. 
con el que Bernardino transfigura míticamente su villa en el monte Echia, en Pizzofalcone ${ }^{22}$, para trasladarse a Mergellina, donde Sannazaro tenía su morada, y así poder seguir sus huellas en lo que podría ser una translatio de género: el movimiento de los «orti» hasta il «sasso» (v. 1) del «lido» (v. 25) en el que se encuentran las ninfas; con ello pretende unir su voz a la del poeta y así llenar la suya de "novi accenti» (v. 26). Así que si, por un lado, cabría suponer que el poeta ha empezado una nueva trayectoria en su poesía, como es introducir la piscatoria en la lengua toscana; por otro lado, el hecho de que quiera revestir su voz de nuevos acentos, la «dolcezzd» y la «meraviglia» (v. 11) sannazarianas, haría pensar que se trataba de una empresa que el poeta había ya iniciado y que ahora quería perfeccionar. Aun así, en el conjunto del libro, que está articulado a la manera del Canzoniere de Petrarca por cuanto tiene su propia historia, la primera égloga cumpliría esta función proemial en la que el poeta dejaría claras cuáles son sus intenciones: imitar las piscatorias de Sannazaro.

De un modo parecido, la égloga IX empieza con una llamada a la propia ninfa para que descienda en lo que supone también una translatio de género, el movimiento de su «bel colle», la poesía bucólica, a la «marina» (v. 1) de las piscatorias. En este caso, a diferencia del anterior, no es el propio personaje el encargado de llevar a cabo este movimiento que antes suponía abandonar la villa de Echia y trasladarse a Mergellina. Aquí es la propia ninfa del poeta la que debe dejar "gli orti ov'Amor vive» (v. 2) puesto que "convien solcar l'onda marina, I e cercar altre piagge et altre rive / con altri remi e con più destro legno» (vv. 4-6). Los «altri remi» son los «toschi remi» que aparecen en la égloga VIII (v. 96), lo que significaría, en contraste con la égloga primera, donde el pescador-poeta buscaba llenar su voz de acentos sannazarianos, que en esta otra égloga lo que se está barajando en realidad es dar comienzo a una nueva empresa: adaptar la piscatoria latina a la lengua toscana.

Este hecho, en consonancia con lo expuesto por Pintor, nos haría pensar que es, dentro de este ambiente cortesano de la isla, donde se compuso la primera de sus piscatorias. Como se ha señalado antes, la égloga celebra el natalicio de Vittoria Colonna. Mientras Egla desciende de la colina y, de alguna manera, le allana el camino: le muestra "libero e piano» "l'ondoso campo" y el "umido sentiero" (vv. 7-8), el poeta hace los preparativos que suponen este homenaje:

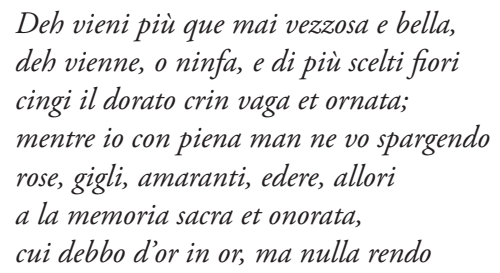

22. «Vi piaceranno poi, credo, per lo riscontro che farete del Rota col Sannazaro, si perché diversamente hanno amendue trattato una stessa materia non solo nella lingua ma nella cosa e ne' concetti, e sì perché vi ricorderete aver l'uno scritto le latine a Mergillina e l'altro le volgari ad Echia, ove sono i suoi bellissimi orti, e per ciò da lui sotto nome di ninfa Egla chiamata» (Scipione Ammirato a Giovan Francesco Mormile; en Berardino Rota, op. cit., pp. 75-76). 
del dì natal di Nice, a cui le Muse
sono compagne e d'or in or più larga
versa l'onda Permesso alma e felice;
a cui servon le Circi e le Meduse,
a cui par ch'Elicona e vesta e sparga
di novelli laureti ogni pendice

(vv. 12-24).

Algo que llama poderosamente la atención es el carácter dramático de la piscatoria; en efecto, el poeta, que hace de voz narradora, es el encargado de acondicionar el espacio antes de que se produzca el diálogo entre Meliseo y Timeta, lo que haría pensar que es posible que se tratase de una égloga destinada a ser representada. Una vez se han dispuesto todos los preparativos, el poema aborda lo que «a Meliseo / disse Timeta, e quel che poi cantâro / insieme sotto il monte que nasconde / percosso il temerario empio Tifeo, / asilo de le Muse illustre e caro» (vv. 25-29); es decir, Ischia. Los dos personajes, dentro de este ambiente, son los que celebran el nacimiento de Vittoria Colonna. El hecho de que uno de ellos se llame Meliseo podría ser una referencia al culto pontaniano existente entre los poetas que integraban el cenáculo de Ischia ${ }^{23}$.

Conviene que nos detengamos ahora en esos primeros versos en los que el poeta llama a la ninfa Egla para que descienda de su «bel colle»:

Scendi dal tuo bel colle a la marina,

o Egla, e lascia gli orti ov'Amor vive.

Creemos que no se ha hecho hincapié en lo que supone, como decíamos, un cambio de género, motivado por el movimiento de la montańa al mar, ni tampoco en el juego intertextual que entraña tal movimiento alegórico. Berardino está parafraseando en realidad toda una serie de versos en los que el propio Sannazaro se atribuye el papel de inventor de la piscatoria o en el que son otros poetas, como Ariosto, los que vienen a hacer esto mismo:

Ipse olim dedit, hanc pastor Melisaeus, ab alta cum me forte senex audisset rupe canentem, et dixit, "Puer, ista tuae sint praemia Musae, quandoquidem nostra cecinisti primus in acta»

(Sannazaro, égloga II, vv. 42-45).

Ipse canam; nunc litoream ne despice Musam quam tibi post silvas, post horrida lustra Lycaei (si quid id est) salsas deduxi primus ad undas

23. Un reflejo de tal culto pontaniano lo tenemos también en el ya citado Dialogo de Giovio, en la estatua que aparece en la descripción del espacio del libro segundo, delante de la cual los personajes hablan de literatura. En opinión de Minonzio (CVII), dicha estatua tendría un doble significado: un significado, por así decir, real, que tendría que ver con los poetas pontanianos que acudían a la isla y que formaban parte del cenáculo (por ejemplo, el propio Berardino Rota), pero también un significado simbólico, común a todos ellos y a su admiración por el célebre poeta humanista. 
ausus inexperta tentare pericula cymba $a^{24}$

(Sannazaro, égloga IV, vv. 17-20).

\section{Quandoquidem salsas descendi ego primus ad undas ausus inexpertis reddere verba sonis}

(Sannazaro, elegía II, lib. III, vv. 57-58).

\section{Iacopo Sanazar che alle Camene}

lasciar fa i monti et habitar l'arene

(Ariosto, Orlando 1516, XL, 9).

Tales versos se remontan en última instancia a la figura del maestro Pontano y al quinto de los Idilios de Teócrito. En la «Lepidina» hay un momento en el que, al referirse a la ninfa Sarnítide, durante el segundo cortejo se nos dice que su poder amoroso y erótico es tal que ha hecho entristecer a los sátiros; entristecer a las napeas; hacer que se consuma el octogenario Alcón; volver loco a Morfeo y, en contra de toda natura, hacer que Fauno abandone los bosques para irse a vivir a la vera del mar: "Deseruit siluas, qui nunc colit aequora, Faunus» (v. 150) ${ }^{25}$; un verso cuya importancia no escapó a Vera Tufano, quien ha dicho, con acierto, tratarse "di un verso densissimo», por el cual

dimensione bucolica e dimensione marina vengono messe consapevolmente in forte contrapposizione. Il soggetto viene ritardato il piu possibile per sortire l'effetto di aprosdoketon a fine del verso. Il baricentro del mondo bucolico nella persona di uno dei suoi rappresentati più illustri viene radicalmente invertito, passando sorprendentemente dai boschi al mare. La tensione fra il mondo marino e quello bucolico, che serpeggia in tutta la Lepidina e che viene generata dall'introduzione innovativa del mare nel mondo pastorale, trova qui quasi un'esplicita istituzionalizzazione. ${ }^{26}$

Esta fusión entre el espacio simbólico del mundo bucólico y del marino, propiciada por el movimiento migratorio del Fauno al mar, tendría su origen en la mención que uno de los dos personajes que protagonizan el $\mathrm{V}$ idilio de

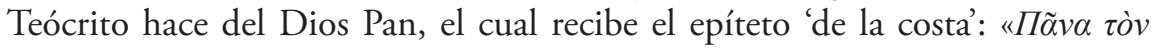

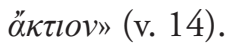

Es posible que Berardino Rota tomara este movimiento de la montańa al mar como forma de atribuirse él también, gracias a este juego intertextual, la primacía dentro del género, en su caso, en la lengua toscana. Es lo que hace también en otras églogas que componen el libro; por ejemplo, en la ya citada égloga VIII, en la que el poeta recogía il "pregio» de la «rete toscana» que le había concedido Vittoria Colonna de un modo parecido a Sannazaro y a las lanas que Meliseus le entrega como premio por haber sido el primero en componer

24. Cf. Horacio, Carm. 3.30, 10-14: «Dicar, qua violens obstrepit Aufidus / et qua pauper aquae Daunus agrestium / regnavit populorum, ex humili potens, / princeps Aeolium carmen ad Italos / deduxisse modos...".

25. Giovanni Pontano, Eclogae, ed., trad. y notas Liliana Monti Sabia, Napoli, Liguori, 1973, p. 33 .

26. Carmela Vera Tufano, Le Eclogae di Giovanni Pontano, tesis de doctorado, Università degli Studi di Napoli Federico II, 2010, p. 82. 
piscatorias; o como hace también, poco más adelante, al autoproclamarse, esta vez sin tapujos, el "primiero» que "il nostro mar con toschi remi» "fende» "e pesca e nuota in disusati modi» (vv. 96-97), paráfrasis, en este caso, de la elegía II sannazariana.

Se trata de un movimiento descendiente que Berardino Rota pudo haber tomado de la elegía segunda, dedicada a Cassandra Marchese; ya que en la égloga cuarta Sannazaro habla de ir más allá de los bosques y de los «horrida lustra» de Liceo para intentar el peligro de navegar con barca inexperta; un movimiento que, en todo caso, no implica la inclinación de la segunda elegía. Por otro lado, la mención de la montaña, y no del bosque, que es la imagen que aparece en Sannazaro, pudo haber sido el fruto de la intermediación de esos versos del Orlando en los que Ariosto atribuye al poeta napolitano el papel de inventor de la piscatoria; en su caso, también hablando de ninfas según la denominación romana, las «Camene».

Reforzaría esta lectura alegórica de los versos de Berardino Rota la presencia de la ninfa Egla ${ }^{27}$, que, si bien, según se ha advertido, es una transfiguración mítica de la villa que el poeta poseía en el monte Echia, por otro lado, algunas referencias que aparecen en el libro nos invitan a pensar que pudiera tratarse de un recuerdo virgiliano; en particular, de la ninfa Egle, el único personaje femenino que aparece representado en las Bucólicas. Un indicio lo tenemos en la égloga $\mathrm{V}$ "Galatea, over Magia», que se inspira en los modelos de la égloga $\mathrm{V}$ sannazariana y del segundo idilio de Teócrito. En este caso, Mopso le dice a Meri lo mismo que

Simeta il disse ad Egla: Egla che fue
non men dotta che bella; Egla mia poi
me l'insegnò, quando io la nassa a pena
alzar potea sul legno, et ambedue
ad Erpili fûr mastre, che co' suoi
incantesmi stupir fe' quest'arena

(vv. 73-78).

El poeta, al adaptar el nombre de Echia, donde tenía su villa, habría tomado una morfología parecida a la del personaje virgiliano, que aquí aparece representado en forma de maga en tanto discípula de la Simeta de Teócrito (idilio II) y maestra de la Herpilis sannazariana (égloga V). Prueba de ello es la manera en la que aparece caracterizado el personaje. Se nos dice, mediante epanalepsis, que es una ninfa bella; del mismo modo que en Virgilio ${ }^{28}$ :

\section{Addit se sociam timidisque superuenit Aegle, Aegle Naiadum pulcherrima, iamque uidenti sanguineis frontem moris et tempora pingit}

(bucólica VI, vv. 20-22).

27. La ninfa aparece también en los Carmina, op. cit, Elegiarum libris 1.6, 11; 2.2, 17, entre otros (cf. la ed. de Cristina Zampese).

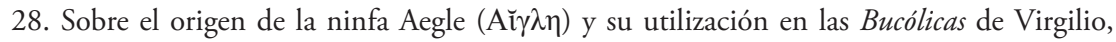
cf. la entrada correspondiente en la Enciclopedia virgiliana (Istituto della Enciclopedia italiana, 1984-1991), s.v., «Egle». 
Por otro lado, también Sannazaro en la quinta de sus piscatorias «Herpylis Pharmaceutria» representaba a Egle en vestes de maga empleando también la anadiplosis y calificándola en su caso de «docta Aegle» (v. 66 ${ }^{29}$; un hecho que podría llevarnos a pensar que Berardino Rota hubiera asimilado para su ninfa estas dos cualidades que, respectivamente, se encontraban ya en Virgilio y en Sannazaro.

Así pues, dentro de este desplazamiento de la montańa al mar, el nombre de Egla, que es quien lo encarna, está plagado de resonancias bucólicas, lo que contribuiría a una mayor significación del pasaje. Curiosamente, también en el Genealogia Deorum Gentilium IV, 68 aparece, vinculado a la gigantomaquia y al mito de Tifeo, símbolo de Ischia, una tal Egla que se la designa como la mujer de Pan. Explica Boccaccio que, según el relato de Teodoncio, una de sus principales fuentes ${ }^{30}$, los gigantes, que nacieron de la sangre derramada en la tierra por los titanes, igual que sus padres movieron guerra contra Júpiter. Ahora bien, para no caer en su mismo error antes tomaron ciertas precauciones, como fue raptar a la también bellísima Egla y dejarla encerrada en una cueva custodiada por la madre de ellos (Gea):

Hos Theodontius dicit pedes anguium habuisse et bellum movisse Iovi, ut patres fecerant; verum nil ausus, donec Egla speciosissima mulierum et coniunx Panis a Terra matre eorum celaretur in specu. ${ }^{31}$

El problema de la explicación de Boccaccio es que alterna los relatos de Ovidio y de Teodoncio, que son prácticamente idénticos a excepción, por un lado, de la figura de Egla, que solo aparece en el relato de este último, y también de los protagonistas de los mismos, que en el caso de Ovidio es Tifeo, mientras en Teodoncio se habla de gigantes en general, sin incluir, en principio, al que se encuentra enterrado bajo la isla de Ischia. A pesar de esta circunstancia, no cabe descartar la hipótesis de que Berardino Rota, al punto de escribir la égloga IX, que tiene como escenario la isla de Ischia, se inspirara en esta otra versión de la leyenda y, además de utilizar a Egla como forma de significar indirectamente su villa en el monte Echia y el personaje virgiliano, quisiera recordar a la mujer de Pan a la que habían encerrado los gigantes que quisieron asaltar el cielo, uno de los cuales se encontraba enterrado en Ischia. También Sannazaro en un pasaje de la Arcadia había incluido a Tifeo dentro del grupo de los gigantes:

29. El personaje aparece también en los epigramas de Sannazaro 1.60 y 2.11, en este caso como destinataria de los mismos.

30. Sobre la identidad desconocida de Teodoncio, cf. las páginas que Álvarez e Iglesias dedican en la traducción castellana de la obra, donde se recogen las principales hipótesis: Giovanni Boccaccio, Genealogía de los dioses paganos, Madrid, Centro de Lingüística Aplicada Atenea, 2008, pp. LX-LXI.

31. Giovanni Bocaccio, Genealogy of the pagan gods, ed., trad. y notas Jon Solomon, Cambridge, The I Tatti Renaissance, 2011, vol. I, p. 600. 
Le pene de fulminati Giganti, che volsero assalire il cielo, son di questo cagione; i quali, oppressi da gravissime montagne, spirano ancora il celeste foco con che furono consumati. Onde adviene che, sì come in altre parti le caverne abondano di liquide acque, in queste ardeno sempre di vive fiamme. E se non che io temo che forse troppo spavento prenderesti, io ti farei vedere il superbo Encelado disteso sotto la gran Trinacria eruttar foco per le rotture di Mongibello; e similmente la ardente fucina di Vulcano, ove li ignudi Ciclopi sovra le sonanti ancudini batteno i tuoni a Giove; et appresso poi sotto la famosa Enaria, la quale voi mortali chiamate Ischia, ti mostrarei il furioso Tifeo, dal quale le estuanti acque di Baia e i vostri monti del solfo prendono il lor calore. ${ }^{32}$

Hasta aquí, recuperando las palabras de Scipione Ammirato, hemos podido observar que más o menos en estos años que siguen a la publicación de las piscatorias sannazarianas, en los que Vittoria Colonna dirige la única carta que se conserva de su relación con Berardino Rota, el poeta napolitano habría compuesto algunas de sus piscatorias dentro del ambiente ischitano que se prefigura en la égloga IX. Los versos iniciales de esta composición mostraban, por un lado, la voluntad con la que el poeta habría querido homenajear la isla recuperando una antigua versión del mito de Tifeo, que significaba también lo bucólico por cuanto estaba protagonizado por la mujer de Pan; y, por otro lado, la carga de reminiscencias virgilianas con las que Rota habría querido acentuar su paso hacia la bucólica marina.

El otro personaje que aparece en la hipótesis de Pintor vinculado a la figura de Berardino Rota y quien de alguna manera vendría a completar la tríada establecida con la marquesa de Pescara es Bernardo Tasso, cuyas piscatorias, en teoría, habrían competido en Ischia con las del poeta napolitano. Recientemente se ha demostrado que es probablemente en 1533 -el año en que Scipione Ammirato afirma que Berardino Rota, en muy poco tiempo, compuso sus piscatorias (o al menos, algunas de ellas)-, cuando Bernardo Tasso estuvo en la isla ${ }^{33}$. Durante este tiempo se sitúan algunas de las composiciones que aparecen en su segundo libro de los Amori, que se escribió entre 1531 y 1534. Ya advertimos en otro lugar acerca de la cuestión nada irrelevante de que la mayoría de los poemas que se contienen en este libro, los que son de tipo cortesano, aparecen vinculados de un modo bastante sospechoso a los miembros de la familia Ávalos-Colonna; en especial a la marquesa de Pescara $y$, en segundo lugar, a Alfonso d'Ávalos ${ }^{34}$. Frente a los escasos poemas que dedica al príncipe de Salerno, a quien teóricamente Tasso entró a servir en el año 1532, destaca en cambio la cantidad de poemas que el poeta dirige a los primeros; unido este hecho al contenido de algunos de ellos y a que Bernardo Tasso reconocía haber estado al servicio de d’Ávalos, nos llevó a formular la hipótesis de que fuera probablemente durante este tiempo, en el año 1533 en que el poeta se encontraba en la isla, cuando acudiera allí como cortesano del marqués del Vasto y cuando hubiera escrito algunos de estos poemas, incluidos

32. Iacopo Sannazaro, Arcadia, ed., introd. y notas Carlo Vecce, Roma, Carocci, 2013, pp. 297-298.

33. Cf. De la Torre Ávalos, op. cit.

34. Ibid., p. 374 y ss. 
también los poemas piscatorios, una hipótesis que recordamos ahora por estar en sintonía con la hipótesis de Pintor y la afirmación de Scipione Ammirato.

No es extraño pues que la protagonista y el escenario de los dos poemas piscatorios que Bernardo Tasso incluye en su segundo libro de los Amori sean los mismos que aparecen en la égloga IX de Berardino Rota ${ }^{35}$. La égloga que Tasso dedica a Vittoria Colonna, junto con el total de églogas y elegías que componen esta su última sección del poemario ${ }^{36}$, tiene también como protagonista a la marquesa de Pescara y como fondo la isla de Ischia. En ella, la marquesa aparece representada míticamente bajo la figura de Crocale, que es el nombre que recibe también en otras composiciones que aparecen en el libro (LXIV, CV). Se trata de un poema que guarda relación con el contenido de la égloga tercera, donde Crocale llora la muerte de su difunto Davalo, el marqués de Pescara. Se ubica, por tanto, dentro de ese periodo de duelo que Vittoria Colonna atravesó en la isla y que la retuvo allí entre 1527 y principios de 1534. El tono elegíaco que la caracteriza y distingue de la égloga de Berardino Rota, acentuado con el paisaje crepuscular en el que tiene lugar la acción, explica que, dentro del conjunto de églogas y elegías, sirva de puente entre unas y otras, al reunir ambas condiciones. Empieza con una imagen de la marquesa, afligida, que desciende del castillo a la costa para hablarles a las olas, «squarciarsi dolente il crine e'l seno, / e dolersi de' fati, e de la morte» (vv. 4-5); hasta que, de pronto, tras escuchar sus lamentos, aparece Galatea, quien «la voce amata / conobbe, e la cagion del suo languire» (vv. 33-34). Galatea le dice a Crocale que ponga fin a su llanto: "rasserena il viso, / e seco il tuo dolor abbino gli anni. / Nereo mio padre, di quest'ampie arene, I di quest'onde Signore, ha 'I cor conquiso I da la tua gran beltà, Nereo figliolo de l' Occean, del gran padre Occeano» (vv. 63-68). También aquí, como podemos observar, Vittoria aparece representada como señora del mar Tirreno; en este caso, no por un dominio explícito de las aguas, sino por ser la amada de Nereo, cuya hija Galatea trata de convencerla de que se case con él. Como no podía ser de otra manera, habida cuenta de los pretendientes a los que Vittoria Colonna rechazó tras la muerte de su marido, también en este caso la marquesa rechaza al hijo del Oceano y con él todas sus posesiones:

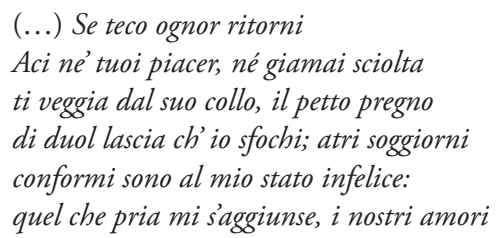

35. Se cita a partir de la edición Bernardo Tasso, Rime, eds. D. Chiodo y V. Martignone, Torino, Res, 1995, vol. 1.

36. Cf. la carta dedicatoria que abre el conjunto de églogas y elegías. Se conservan otras cinco cartas de la relación entre Bernardo Tasso y Vittoria Colonna. Estas se encuentran en la edición Giglio 1559: cuatro en el libro primero, que son las mismas que se contienen en el Carteggio de Ferrero y Müller; más una quinta que aparece en el tercer libro. Hay también una última, no conservada, a la que se refiere la propia marquesa en una misiva que dirige a Bembo (cf. Carteggio, p. 271), si es que detrás del «M. Bernardo» al que se alude se halla la figura de Tasso. 
sen portò seco; i miei beati giorni

quel se gli abbi e ne goda in ciel felice

(vv. 102-109).

Resignada ella, el poema termina como empieza:

In questa Apollo al bel nostro orizzonte
tolse la luce, e già con le fosc'ali
copria la notte il cerchio de la terra,
onde ritorno fe' Crocale al monte
accompagnata da' suoi lunghi mali,
a l'acque Galatea salse e fatali

(vv. 116-121).

Siguiendo su teoría de que Vittoria Colonna habría patrocinado en cierto modo el nacimiento de la piscatoria en vulgar -una teoría que se vería respaldada por la manera en que ambos poetas representan a Vittoria como una ninfa con poderes marinos-, Pintor esgrime algunos argumentos por los que Berardino Rota, y no Bernardo Tasso, habría merecido «il pregio» de la marquesa de Pescara $^{37}$. Creemos que tales argumentos merecen ser tenidos en consideración no solo porque nos parece que se ajustan a la realidad y aúnan la obra de los dos escritores, sino porque subrayan también su carácter altamente experimental: explícito, en el caso de la piscatoria rotiana, como puede apreciarse en esa suerte de reflexión metapoética inicial a la que antes nos referíamos; y solo intuido en la piscatoria de Bernardo Tasso. Por otro lado, es dentro de Ischia donde, según hemos tenido ocasión de ver, se habría fraguado, a imitación del Cortesano de Castiglione, el Dialogo de Giovio, y, donde, nada impide pensar, como afirma también el erudito, hubiera tenido su origen la piscatoria en lengua vulgar.

Pintor compara el ejemplo de la égloga tassiana con la de su modelo en Sannazaro con el fin de medir la distancia que la separa de la égloga de Berardino Rota, que parece ceñirse más al género. Uno de los aspectos que salta a la vista ya de buenas a primeras, y que quizá explique las demás diferencias, es la escasa presencia del modelo sannazariano y de sus fuentes en Virgilio y Teócrito. Si en las églogas de Berardino Rota, como hemos visto, su presencia era constante, incluso al punto de atribuirse el papel de introductor de la piscatoria, parece que en la égloga tassiana pese muchísimo más la obra poética de Vittoria Colonna, cuyo eje central, como sabemos, lo constituye, al menos en una primera parte, la alabanza del marido muerto y su manera íntima de encarar la muerte; de ahí su carácter más elegíaco que amoroso, que es lo que caracteriza en general la égloga sannazariana y en particular a sus piscatorias. La distancia con el modelo supone también la falta de vivacidad en el paisaje, en el que los elementos marinos, en consonancia con el esquematismo general de la composición, aparecen, más que integrados, casi catalogados en los dones que Nereo le promete a su amada Crocale. A nuestro modo de ver, este es un indicio de que Bernardo Tasso estaba más preocupado por el desarrollo de la 
acción, que a la postre resulta simple, que en los pequeños detalles que, en teoría, habrían de revestirla, a diferencia de las piscatorias sannazarianas o del elevado despliegue de medios que muestra la «Lepidina» del maestro Pontano. Este alejamiento con respecto al modelo explicaría, en suma, que ni siquiera los protagonistas de la égloga sean pescadores; una «lectura superficial», como la llama Pintor, que se hallaría también presente en su otra composición piscatoria.

En las mismas fechas en que Tasso escribió la égloga número VII, también habría compuesto el poema que podríamos catalogar de "soneto piscatorio", el número LXIV de la colección. El hecho de que se trate de un soneto y no de una égloga, como sucedía en el poema anterior, vendría de alguna manera a reforzar la idea de que se estaba experimentando con la piscatoria neolatina, adaptándola incluso a otros metros. El asunto es idéntico al de la égloga, aunque en este caso asuma la voz poética el enamorado de Crocale. Nereo le pide a la ninfa que abandone su encierro en el monte de Enaria (Ischia) para que le muestre sus bellezas «eterne e conte». Se trata también de un poema sin pescadores cuyas referencias principales al mundo marino se reducen, en este caso, a la escenificación costera en que se produce la acción y a la presencia del dios marino que implora a Crocale; de ahí que lo más interesante y cuanto quisiéramos destacar sea únicamente la relación temática que este poema mantiene con el anterior y que lo situaría también dentro de los poemas piscatorios que Bernardo Tasso escribió en Ischia.

Estas son las dos únicas composiciones piscatorias que aparecen en el segundo libro de los Amori. Al comienzo del quinto libro de las Rime di diversi illustri signori napoletani (Giolito 1552), entre varios sonetos que Alfonso d'Ávalos escribe, encontramos uno que quisiéramos reproducir en su integridad dado el interés que presenta para el contenido de este trabajo ${ }^{38}$ :

\author{
In mezzo a l'onde salse in fragil legno \\ un pescator vid'io d'età novella, \\ a cui il fior novo per la guancia bella \\ fatto ancor non avea pur picciol segno. \\ Egli adoprava ogni sua forza e ingegno \\ per gir in porto, e fuggir la procella \\ che dietro lo seguia con questa e quella \\ onda, mostrando ogn'hor più fero sdegno. \\ "Ecco i pesci chio tolsi, ti ritorno; \\ la rete mia ti dono, e non mè grave»: \\ cosi con humil voce al mar dicea.
}

38. El soneto se encuentra en la página 6. Sobre las Rime di diversi illustri signori napoletani, cf. el estudio de Toscano, «Le Rime di diversi illustri signori napoletani: preliminari d'indagine su una fortunata antologia", en Letterati corti accademie. La letteratura a Napoli nella prima metà del Cinquecento, Napoli, Loffredo, 2000, cap. 6, pp. 183-200. 
Allor allor si fe' sereno il giorno,

l'onde tranquille, e'l vento aura soave;

$e$ in braccio nel raccolse Galatea. ${ }^{39}$

Este poema aparece también recogido dos décadas más tarde en el vigesimoquinto volumen del Parnaso italiano, el cual lleva por título Marittimi e pedanteschi y reúne la obra de Bernardino Baldi, Berardino Rota, Fidentio, Niccolò Franco y el marqués del Vasto ${ }^{40}$. Casi todas tienen el denominador común de que se trata de composiciones que, de una u otra manera, se relacionan con el mar; sin embargo, unas entrarían dentro de la categoría de piscatorias y otras de poemas «marittimi» o "marinareschi». A simple vista, la principal diferencia entre unos y otros es que las piscatorias se sitúan en un paisaje litoral y, en principio, tienen como protagonistas a pescadores, mientras que los poemas «marinarescos» se desarrollan en medio del mar y sus protagonistas son marineros. Andrea Rubbi, quien prologa el volumen y lleva a cabo la selección de este Parnaso, sitúa el poema de Alfonso d'Ávalos junto con los sonetos de Niccolò Franco dentro del grupo de los «sonetti marinareschi»:

Vidi la Piscatoria del conte di S. Martino, opera mista di prosa e verso, ma non l'ho creduta degna di voi. Scorsi l'egloghe di Andrea Calmo, e di Giulio Cesare Capaccio, e in fine ho deciso che la Nautica del Baldi, e la Piscatoria del Rota siano soli quei due poemi che possono aver luogo nel nostro Parnaso. Non escluso però alcuni sonetti marinareschi di Niccolò Franco, e del marchese del Vasto. ${ }^{41}$

Ahora bien, si echamos una ojeada al contenido de este poema, rápidamente nos damos cuenta de que parece incluir elementos de ambas clases. Si por un lado, el protagonista se encuentra en medio de las aguas sobre una barca frágil que simboliza su propia fragilidad ante el tormentoso mar, que es Galatea; por el otro, el hecho de que se trate de un pescador, cuya red y su pesca abandona por la ninfa, nos haría pensar que también pudiera inscribirse dentro del género piscatorio, en una suerte de composición mixta entre la piscatoria y el poema marítimo.

Sabemos que en 1533, cuando Tasso y Berardino Rota componen en teoría sus poemas piscatorios, Alfonso d'Ávalos se encontraba en Ischia por un problema de salud que le retuvo allí durante varios meses: sus continuos achaques de gota ${ }^{42}$. Si bien es cierto que, a diferencia de lo que sucede en las otras composiciones, los personajes del poema no están vinculados a los demás miembros de la casa Ávalos-Colonna, no cabe descartar que también hubiera podido concebirse en el mismo tiempo que los anteriores; contribuiría a esta

39. Se ha modernizado la puntuación y las grafías.

40. Baldi, Rota, Franco, del Vasto, Fidentio, Marittimi e pedanteschi del secolo XVI, en Parnaso italiano ovvero raccolta dé poeti classici italiani, Venezia, Antonio Zatta e figli, 1787, t. XXV, p. 208.

41. Ibid. Cf. la introducción "A' suoi amici».

42. Cf. De la Torre Ávalos, op. cit., pp. 379-380; Gennaro Varriale, «Nápoles y el azar de Corón (1532-1534)», Tiempos modernos, vol. 7, n 22, 2011, pp. 20-21. 
idea la tipología de su protagonista y también la figura de Alfonso d'Ávalos, quien, de ser cierta la hipótesis de Pintor, habría sido testigo de la justa literaria entre Bernardo Tasso y Berardino Rota.

A modo de conclusión: no nos es posible afirmar que en 1533 Bernardino Rota escribiera en Ischia sus piscatorias, a no ser que demos por válidas, sin más, las palabras de Scipione Ammirato. Ahora bien, el hecho de que tanto Alfonso d'Ávalos como Bernardo Tasso estuvieran presentes en la isla durante el tiempo en que teóricamente Rota escribió algunas de sus piscatorias, o por lo menos la égloga IX, unido este hecho al contenido de las piscatorias tassianas, con toda claridad relacionadas por su asunto con el cenáculo de Ischia, supondrían algunos indicios no solo concordantes con la afirmación de Ammirato sino también con la hipótesis de Pintor. Faltaría alguna evidencia, por ejemplo alguna carta, que sirviera para demostrar que fue en Ischia donde se gestó la piscatoria en lengua vulgar, aunque ello sea a todas luces lo más probable. 